Proceedings of the 42th "Jaszowiec" International School and Conference on the Physics of Semiconductors, Wisła 2013

\title{
PTCDI-C8 Adsorption on Si(100)
}

\author{
K. Lament, P. Mazur, S. Zuber and A. Ciszewski \\ Institute of Experimental Physics, Faculty of Physics and Astronomy, University of Wrocław \\ pl. M. Borna 9, 50-204 Wrocław, Poland
}

\begin{abstract}
Adsorption of $N, N^{\prime}$-dioctyl-3,4,9,10-perylenedicarboximide (PTCDI-C8) on the $\mathrm{Si}(100)$ surface has been examined using X-ray photoelectron spectroscopy and scanning tunneling microscopy. X-ray photoelectron spectroscopy results show that the bonds are formed between the carbonyl groups of the molecules and the substrate. Scanning tunneling microscopy results show that the first and further layers are disordered.
\end{abstract}

DOI: 10.12693/APhysPolA.124.775

PACS: 81.07.Pr, 79.60.-i, 68.37.Ef

\section{Introduction}

Hybrid materials consisting of organic molecules and conventional inorganic semiconductors are important from the point of view of modern electronics (OLEDs, organic thin-film transistors, solar cells, etc.). However, understanding the mechanisms of growth and self-organization of molecules on inorganic substrates is still a problem. Thus it is important to understand moleculesubstrate and molecule-molecule interactions and their impact on the formation of the first layer of ordered film.

There are known results of adsorption of perylene derivatives, such as PTCDA and DiMe-PTCDI on semiconductors [1-4]. These results indicate that, under certain conditions, it is possible to obtain organic well-ordered structures.

Using X-ray photoelectron spectroscopy (XPS), and scanning tunneling microscopy (STM), we studied the PTCDI-C8/Si(100) system to obtain information about the mechanisms of adsorption and thin film growth of the molecules on this substrate.

\section{Experiment}

All measurements were carried out under ultrahigh vacuum conditions (UHV) at room temperature in two chambers equipped with an energy analyzer Phoibos 100-5 with magnesium X-ray source, and a scanning tunneling microscope VT STM/AFM Omicron with tungsten tip.

$\mathrm{Si}(100)$ sample (B-doped, resistivity $0.06-0.07 \Omega \mathrm{cm}$ ) was chemically cleaned using the RCA procedure. The sample was annealed for $9 \mathrm{~h}$ at $500^{\circ} \mathrm{C}$, and then flashed several times at temperatures from about 1350 to $1400{ }^{\circ} \mathrm{C}$, which gave as a result a well reconstructed surface. Thin layers of PTCDI-C8 (Sigma-Aldrich) were evaporated in situ at $300^{\circ} \mathrm{C}$ on the substrate kept at room temperature. The WSxM software was used for the analysis of STM data [5].

\section{Results and discussion}

\subsection{Low coverages}

Figure 1 shows XPS results obtained for the $\mathrm{Si}(100)$ surface coated with 0.4 ML of PTCDI-C8 molecules. In the $\mathrm{C} 1 s$ region, as a result of decomposition, four peaks were identified. The least intense peak (1) is related to the presence of carbon atoms on the surface before deposition of the molecules. The most intense peak (2) $(284.8 \mathrm{eV})$ responds to binding between the carbon atoms in the perylene core. The third peak (3) $(285.8 \mathrm{eV})$ corresponds to the $\mathrm{C}-\mathrm{O}-\mathrm{Si}$ bond, which is formed during the reaction of carbonyl groups with the Si surface. Peak (4) $(287.9 \mathrm{eV})$ corresponds to the $\mathrm{C}-\mathrm{N}$ bond of the end-group of molecule. The presence of this peak can be explained in two ways: (a) in addition to the molecules bonded to $\mathrm{Si}$ atoms, on the surface there are also weakly bounded molecules, (b) not all of the oxygen atoms react with $\mathrm{Si}$ atoms, hence part of the end-groups remains unchanged. Similar spectra were obtained for thin films of other perylene derivative, PTCDA, adsorbed on the surface of the $\mathrm{Si}(100)[1-3]$. The same types of bonding to the substrate were identified for PTCDA on $\operatorname{Si}(111)$ [6].

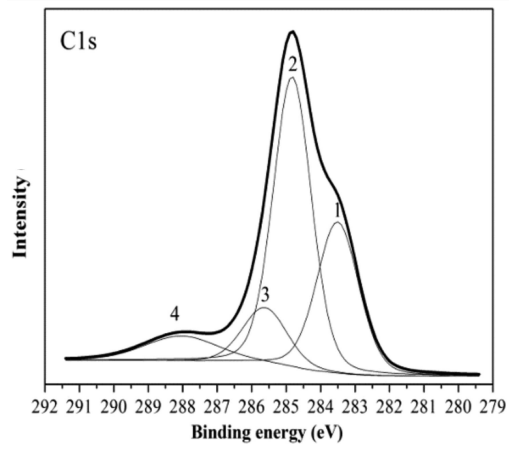

Fig. 1. C $1 \mathrm{~s}$ spectrum of $0.4 \mathrm{ML}$ adsorbed on the $\mathrm{Si}(100)$ surface. Description of the peaks is discussed in the text.

For further information about the process of adsorption of PTCDI-C8 molecules on $\mathrm{Si}(100)$, the system was investigated using STM. Figure 2 shows STM images obtained after deposition of very small amounts of molecules. Adsorbate is visible as a bright protrusions labeled A and B. We observed two types of structures: structure A is almost parallel to the row of Si dimers, while structure B lies asymmetrically to the substrate. Both types of structures are larger than a single molecule 

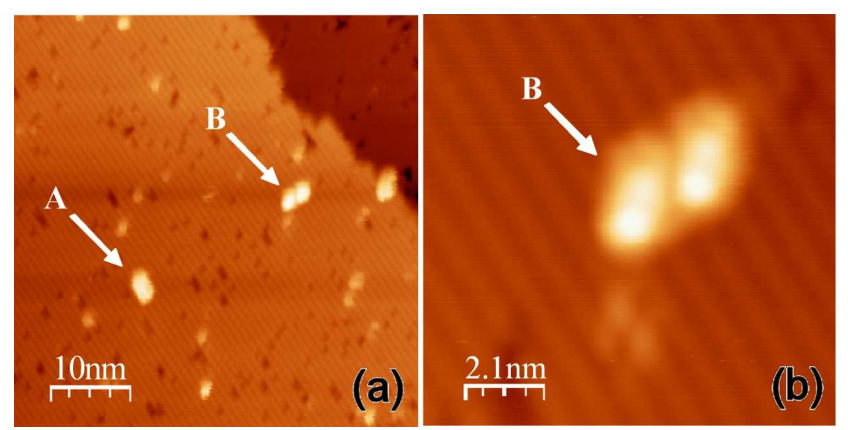

Fig. 2. STM images obtained for low coverages of PTCDI-C8. Two types of agglomerates are shown (A, B). Imaging parameters: (a) $V_{s}=-1.2 \mathrm{~V}, I_{t}=$ $115.4 \mathrm{pA}$; (b) $V_{s}=-1.2 \mathrm{~V}, I_{t}=49.3 \mathrm{pA}$.

(long axis of the core has a length $1.2 \mathrm{~nm}$ ), from where we concluded that the molecules form small agglomerates. Statistics show that $70 \%$ of the B-agglomerates is on the surface . Orientation of the agglomerates in relation to the substrate is random.

\subsection{High coverages}

Figure 3 shows the data in the $\mathrm{C} 1 s$ region obtained for $1 \mathrm{ML}$ of PTCDI-C8. Decomposition of this area is similar to the one presented in Fig. 1, however there are significant changes in the intensities of the individual components. The peak labeled (2) is less intensive than in the spectrum obtained for $0.4 \mathrm{ML}$, and its height is now almost equal to the height of the peak (3). We conclude that the number of molecules bonded to the substrate in this case is comparable with the number of unbonded molecules. We assume the presence of a three-dimensional growth, in which the first well-bonded layer is covered by molecules that are weakly bonded.

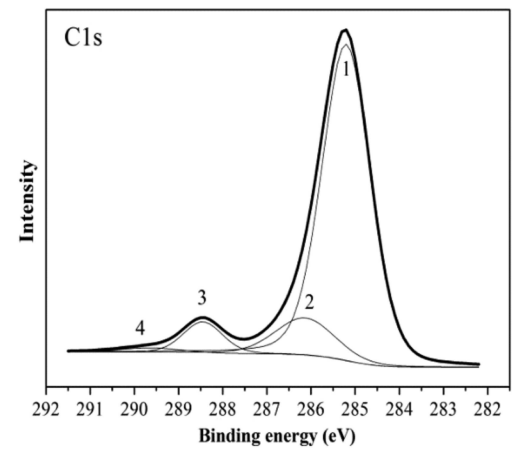

Fig. 3. C $1 s$ spectrum for $1 \mathrm{ML}$ of PTCDI-C8 adsorbed on the $\mathrm{Si}(100)$. Description of the peaks is discussed in the text.

In order to determine the mechanism of growth, the STM was used. Images obtained for a large coverage (Fig. 4) show lack of order in the film. Adsorbate occurs in the form of unevenly distributed islands with different sizes and heights. Considering the presence of dangling bonds on a clean surface, we can assume that the reason for the disorder are the strong adsorbate-substrate interactions that dominate the intermolecular interactions, therefore self-organization is not possible. Furthermore, probable bond of one end-groups, not both, causes that the molecules in the first layer may be flat or stand up on the substrate. Lack of order in the first layer may be the reason for the disorder in the entire film. Figure $4 \mathrm{~b}$ shows that at a positive bias it is possible to obtain images of molecular orbitals, however, because of disordering it is not possible to determine exactly how the molecules are arranged in relation to the substrate and to each other.

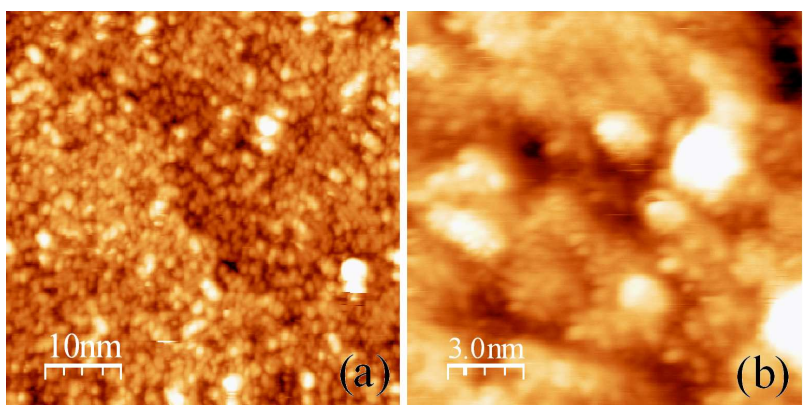

Fig. 4. (a) STM image for high coverage of PTCDI-C8 $\left(V_{s}=-1.7 \mathrm{~V}, I_{t}=101.2 \mathrm{pA}\right)$. (b) Molecular orbitals in disordered layer of PTCDI-C8 are visible only for unoccupied states $\left(V_{s}=0.9 \mathrm{~V}, I_{t}=103.5 \mathrm{pA}\right)$.

\section{Conclusions}

PTCDI-C8 molecules adsorbed on the clean surface of the $\mathrm{Si}(100)$ do not form ordered layers. At low coverage two types of agglomerates with different orientation in relation to the substrate are formed. It was found that the molecules bind to the substrate by a carbonyl group. There are different arrangements of molecules in relation to the substrate, and disordered films are obtained.

\section{References}

[1] T. Soubiron, F. Vaurette, J.P. Nys, B. Grandidier, X. Wallart, D. Stievenard, Surf. Sci. 581, 178 (2005).

[2] J.B. Gustafsson, E. Moons, S.M. Widstrand, L.S.O. Johansson, Surf. Sci. 572, 23 (2004).

[3] J.B. Gustafsson, E. Moons, S.M. Widstrand, M. Gurnett, L.S.O. Johansson, Surf. Sci. 572, 32 (2004).

[4] T.U. Kampen, G. Salvan, A. Paraian, C. Himcinschi, A.Y. Kobitski, M. Friedrich, D.R.T. Zahn, Appl. Surf. Sci. 212, 501 (2003).

[5] I. Horcas, R. Fernandez, J.M. Gomez-Rodriguez, J. Colchero, J. Gomez-Herrero, A.M. Baro, Rev. Sci. Instrum. 78, (2007).

[6] N. Nicoara, O. Ṕaz, J. Méndez, A.M. Baró, J.M. Soler, J.M. Gómez-Rodríguez, Phys. Rev. B 82, 075402 (2010). 